\title{
Deep Gated Recurrent and Convolutional Network Hybrid Model for Univariate Time Series Classification
}

\author{
Nelly Elsayed ${ }^{1}$, Anthony S Maida ${ }^{2}$ \\ School of Computing and Informatics, \\ University of Louisiana at Lafayette, \\ Louisiana, USA
}

\begin{abstract}
Hybrid LSTM-fully convolutional networks (LSTM-FCN) for time series classification have produced stateof-the-art classification results on univariate time series. We empirically show that replacing the LSTM with a gated recurrent unit (GRU) to create a GRU-fully convolutional network hybrid model (GRU-FCN) can offer even better performance on many time series datasets without further changes to the model. Our empirical study showed that the proposed GRU-FCN model also outperforms the state-of-the-art classification performance in many univariate time series datasets without additional supporting algorithms requirement. Furthermore, since the GRU uses simpler architecture than the LSTM, it has fewer training parameters, less training time, smaller memory storage requirements, and simpler hardware implementation, compared to the LSTM-based models.
\end{abstract}

Keywords-GRU-FCN; LSTM; fully convolutional neural network; time series; classification

\section{INTRODUCTION}

A time series (TS) is a sequence of data points obtained at successive equally-spaced time points, ordinarily in a uniform interval time domain [1]. TSs are used in several research and industrial fields where temporal analysis measurements are involved such as in signal processing [2], pattern recognition [3], mathematics [1], psychological and physiological signals analysis [4], [5], earthquake prediction [6], weather readings [7], and statistics [1]. There are two types of time series: univariate and multivariate. In this paper, our objective is to study the univariate time series classification.

There are many approaches to time series classification. The distance-based classifier based on the k-nearest neighbor $(\mathrm{KNN})$ algorithm is considered a baseline technique for time series classification. Mostly, a distance-based classifier uses Euclidean or Dynamic Time Warping (DTW) as a distance measure [8]. Feature-based time series classifiers are also widely used such as the bag-of-SFA-symbols (BOSS) [9] and the bag-of-features framework (TSBF) [10] classifiers. Ensemble-based classifiers combine separate classifiers into one model to reach a higher classification accuracy such as the elastic ensemble (PROP) [11], and the collective of transformbased ensemble (COTE) [12] classifiers.

Convolutional neural network (CNN) based classifiers have advantages over other classification methods because CNNs provide the classifier with a preprocessing mechanism within

\author{
Magdy Bayoumi ${ }^{3}$ \\ Dep. of Electrical and Computer Engineering, \\ University of Louisiana at Lafayette, \\ Louisiana, USA
}

TABLE I. COMPARISON OF GRU AND LSTM COMPUTATIONAL ELEMENTS.

\begin{tabular}{lll} 
Comparison & LSTM & GRU \\
\hline number of gates & 3 & 2 \\
number of activations & 2 & 1 \\
state memory cell & Yes & No \\
number of weight matrices & 8 & 6 \\
number of bias vectors & 3 & 4 \\
number of elementwise multiplies & 3 & 3 \\
number of matrix multiplies & 8 & 6
\end{tabular}

the model. Examples are the multi-channel CNN (MC-CNN) classifier [13], the multi-layered preceptron (MLP) [4], the fully convolutional network (FCN) [4] and, specifically, the residual network (ResNet) [4].

The present paper focuses on the recurrent neural network based classification approaches such as LSTM-FCN [5] and ALSTM-FCN [5] that are the first recurrent-based time series classification models. These models combine both temporal CNNs and long short-term memory (LSTM) models to provide the classifier with both feature extraction and time dependencies through the dataset during the classification process. These models use additional support algorithms such as attention and fine-tuning algorithms to enhance the LSTM learning due to its complex structure and data requirements.

This paper attempts to emerge the difference between the GRU and LSTM in univariate time series classification purpose. This paper studies whether the use of gated-recurrent units (GRUs) can improve the hybrid classifiers listed above with. We create the GRU-FCN by only replacing the LSTM with a GRU in the LSTM-FCN [5]. We intentionally kept the other components of the entire model without changes to make an empirical comparison between the LSTM and GRU in the same model structure to obtain a fair comparison between both architectures regarding the univariate time series classification task. Like the LSTM-FCN, our model does not require feature engineering or data preprocessing before the training or testing stages. The GRU is able to learn the temporal dependencies within the dataset. Moreover, the GRU has a smaller block architecture and shows comparable performance to the LSTM without a need for additional algorithms to support the model.

Although it is difficult to determine the best classifier for all time series types, the proposed model seeks to achieve equivalent accuracy to state-of-the-art classification models 


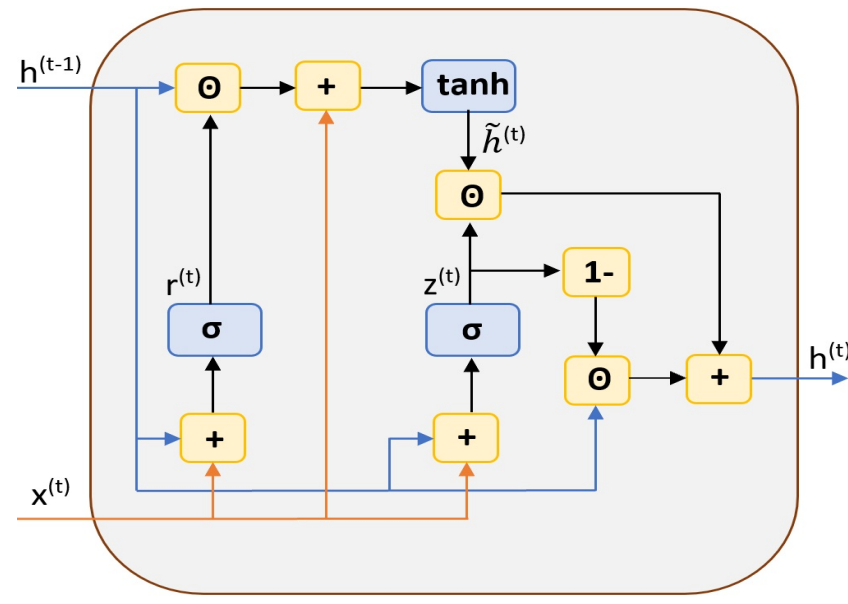

Fig. 1. Block architecture for an unrolled GRU.

in univariate time series classification. Following [4] and [5], our tests use the UCR time series classification archive benchmark [14] to compare our model with other state-ofthe-art univariate time series classification models. Our model achieved higher classification performance on several datasets compared to other state-of-the-art classification models.

\section{Model COMPONENTS}

\section{A. Gated Recurrent Unit (GRU)}

The gated recurrent unit (GRU) was introduced in [15] as another type of gate-based recurrent unit which has a smaller architecture and comparable performance to the LSTM unit. The GRU consists of two gates: reset and update. The architecture of an unrolled GRU block is shown in Fig. 1. $r^{(t)}$ and $z^{(t)}$ denote the values of the reset and update gates at time step $t$, respectively. $x_{i} \in \mathbb{R}^{n}$ is a $1 \mathrm{D}$ input vector to the GRU block at time step $t . \tilde{h}^{(t)}$ is the output candidate of the GRU block. $h^{(t-1)}$ is the recurrent GRU block output of time step $t-1$ and the current output at time $t$ is $h^{(t)}$. Assuming a one-layer GRU, the reset gate, update gate, output candidate, and GRU output are calculated as follows [15]:

$$
\begin{aligned}
& z^{(t)}=\sigma\left(W_{z x} x^{(t)}+U_{z h} h^{(t-1)}+b_{z}\right) \\
& r^{(t)}=\sigma\left(W_{r x} x^{(t)}+U_{r h} h^{(t-1)}+b_{r}\right) \\
& \tilde{h}^{(t)}=\tanh \left(W_{x} x^{(t)}+U_{h}\left(r^{(t)} \odot h^{(t-1)}\right)+b\right) \\
& h^{(t)}=\left(1-z^{(t)}\right) \odot h^{(t-1)}+z^{(t)} \odot \tilde{h}^{(t)}
\end{aligned}
$$

where $W_{z x}, W_{r x}$, and $W_{x}$ are the feedforward weights and $U_{h z}, U_{h r}$, and $U_{h}$ are the recurrent weights of the update gate, reset gate, and output candidate activation respectively. $b_{z}, b_{r}$ and $b$ are the biases of the update gate, reset gate and the output candidate activation $\tilde{h}^{(t)}$, respectively. Fig. 3 shows the GRU architecture with weights and biases made explicit.

Like the RNN and LSTM, the GRU models temporal (sequential) datasets. The GRU uses its previous time step output and current input to calculate the next output. The GRU has the advantage of a smaller size over the LSTM. The GRU consists of two gates (reset and update), while the LSTM has three gates: input, output and forget. The GRU has one unit activation, but the LSTM has two unit activations:

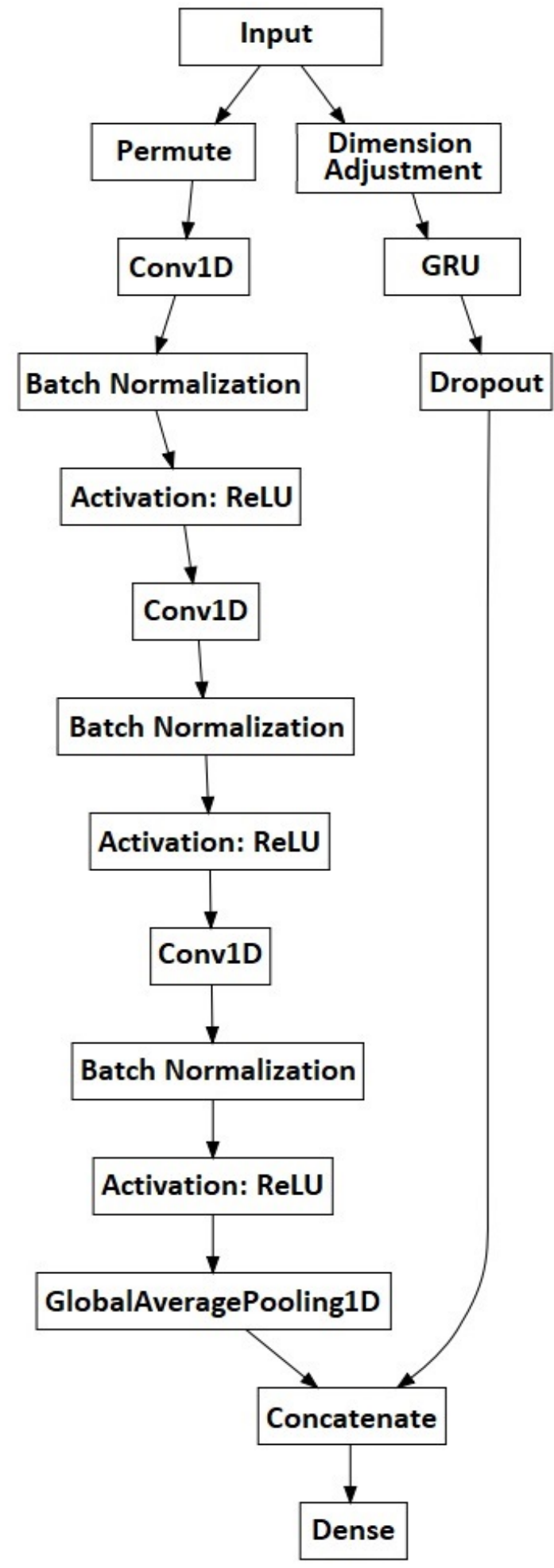

Fig. 2. The proposed GRU-FCN model architecture diagram rendered using the Keras visualization tool and modified from [4], [5] architectures. 


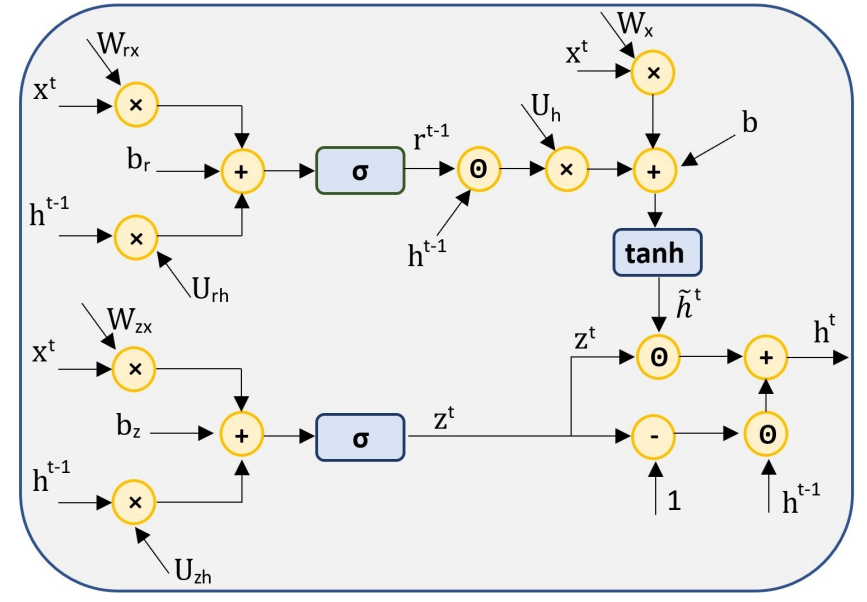

Fig. 3. The GRU architecture showing the weights of each component.

input-update and output activations. Also, the GRU does not contain the memory state cell which exists in the LSTM model. Thus, the GRU requires fewer trainable parameters, and shorter training time compared to the LSTM. Table I compares GRU and LSTM architecture components.

\section{B. Temporal Convolutional Neural Network}

The Convolutional Neural Network (CNN), introduced in 1989 [16], utilizes weight sharing over grid-structured datasets such as images and time series [17], [18]. The convolutional layers within the CNN learn to extract complex feature representations from the data with little or no preprocessing. The temporal FCN consists of many layers of convolutional blocks that may have different or same kernel sizes, followed by a dense layer softmax classifier [18]. For time series problems, the values of each convolutional block in the FCN, are calculated as follows [4]:

$$
\begin{aligned}
& y_{i}=W_{i} * x_{i}+b_{i} \\
& z_{i}=B N(y) \\
& \text { out }_{i}=\operatorname{ReL} U(z)
\end{aligned}
$$

where $x_{i} \in \mathbb{R}^{n}$ is a $1 \mathrm{D}$ input vector which represents a time series segment, $W_{i}$ is the $1 \mathrm{D}$ convolutional kernel of weights, $b_{i}$ is the bias, and $y$ is the output vector of the convolutional block $i . z_{i}$ is the intermediate result after applying batch normalization [19] on the convolutional block which then is passed to the rectified linear unit $R e L U$ [20] to calculate the output of the convolutional layer out ${ }_{i}$.

\section{MODEL ARCHITECTURE}

As stated in the introduction, our model replaces the LSTM with a GRU in a hybrid gated-FCN. We intentionally did not change the other components of the entire model to attain a fair comparison between GRU and LSTM architectures in the same model structure for univariate time series classification. Our model is based on the framework introduced in [4], [5]. The proposed architecture actual implementation is shown in Fig. 2. The architecture has two parallel parts: a GRU and a temporal FCN. Our model uses three-layered FCN architecture proposed in [4]. The dimension adjustment aims to change the dimensions of the input to be compatible with the GRU recurrent design [21]. We also used the global average pooling layer [22] to interpret the classes and to reduce the number of trainable parameters comparing to the fully connected layer, without any sacrifice in the accuracy. The FCN 1D kernel numbers are 128, 256 , and 128 with kernel sizes 8,5 , and 3 in each convolutional layer, respectively. The weights were initialized using the $\mathrm{He}$ uniform variance scaling initializer [23]. In addition, we used the GRU instead of LSTMs that were used in [5] models to reduce the number of trainable parameters, memory, and training time. Moreover, we removed the masking and any extra supporting algorithms such as an attention mechanism, and fine-tuning that were used in the LSTM-FCN and ALSTMFCN models [5]. The GRU is unfolded by eight unfolds as used in [5] for univariate time series. The hyperbolic tangent ( $\tanh$ ) function used as the unit activation and the hard-sigmoid (hardSig) function [24] is used as the recurrent activation (gate activation) of the GRU architecture. The weights were initialized using the glorot_uniform initializer [25], [26] and the biases were initialized to zero. The input was fitted using the concept used in [5] to fit an input to a recurrent unit. We used the Adam optimization function [27] with $\beta_{1}=0.9$, $\beta_{2}=0.999$ and initial learning rate $\alpha=0.01$. The learning rate $\alpha$ was reduced by a factor of 0.8 every 100 training steps until it reached the minimum rate $\alpha=0.0001$. The dense layer uses the softmax classifier [28] using the categorical crossentropy loss function [18]. In this paper, our goal is to make a fair comparison between the LSTM-based model and our GRU-based model. Thus, we used the same number of epochs that were assigned by the original LSTM-FCN model [5] for each univariate time series. The number of epochs that we assigned for each dataset used is shown in Table II.

The input to the model is the raw dataset without applying any normalizations or feature engineering prior to the training process. The FCN is responsible for feature extraction from the time series [4] and the GRU enables the model to learn temporal dependencies within the time series. Therefore the model learns both the features and temporal dependencies to predict the correct class for each training example.

\section{Method And Results}

We implemented our model by modifying the original LSTM-FCN [5]. We found that the fine-tuning algorithm has not been applied in the actual LSTM-FCN and ALSTM-FCN implementation source code on Github which shared by the authors [5] and mentioned in their literature. In addition, the LSTM-FCN [5] authors used a permutation algorithm for fitting the input to the FCN part which was not mentioned in their literature. Therefore, we generated the actual LSTMFCN and ALSTM-FCN implementations to record the results based on their actual code implementation. In addition, to record their training time, memory requirement, the number of parameters and f1-score. The Keras API [26] with TensorFlow backend [29] were used in the implementation of the LSTM-FCN, ALSTM-FCN and GRU-FCN models. The source code of our GRU-FCN implementation can be found on Github: https://github.com/NellyElsayed/GRU-FCN-modelfor-univariate-time-series-classification.

We tested our model on the UCR time series archive [14] as one of the standard benchmarks for time series classification. 
TABLE II. THE UCR DATASETS DESCRIPTIONS BASED ON [14] AND THEIR EXPERIMENTAL ADJUSTMENTS USED IN THE GRU-FCN IMPLEMENTATION.

\begin{tabular}{|c|c|c|c|c|c|c|c|c|}
\hline Dataset & Type & \# Classes & Length & Train size & Test size & \# epochs & $\begin{array}{l}\text { Train } \\
\text { Batch }\end{array}$ & $\begin{array}{l}\text { Test } \\
\text { Batch }\end{array}$ \\
\hline Adiac & Image & 37 & 176 & 390 & 391 & 4000 & 128 & 128 \\
\hline ArrowHead & Image & 3 & 251 & 36 & 175 & 4000 & 32 & 128 \\
\hline Beef & Spectro & 5 & 470 & 30 & 30 & 8000 & 64 & 64 \\
\hline BeetleFly & Image & 2 & 512 & 20 & 20 & 8000 & 64 & 64 \\
\hline BirdChicken & Image & 2 & 512 & 20 & 20 & 8000 & 64 & 64 \\
\hline $\mathrm{Car}$ & Sensor & 4 & 577 & 60 & 60 & 2000 & 128 & 128 \\
\hline $\mathrm{CBF}$ & Simulated & 3 & 128 & 30 & 900 & 2000 & 32 & 128 \\
\hline ChlorineConc & Sensor & 3 & 166 & 467 & 3840 & 2000 & 128 & 128 \\
\hline CinCECGTorso & Sensor & 4 & 1639 & 40 & 1380 & 500 & 128 & 128 \\
\hline Coffee & Spectro & 2 & 286 & 28 & 28 & 500 & 64 & 64 \\
\hline Computers & Device & 2 & 720 & 250 & 250 & 2000 & 128 & 128 \\
\hline CricketX & Motion & 12 & 300 & 390 & 390 & 2000 & 128 & 128 \\
\hline CricketY & Motion & 12 & 300 & 390 & 390 & 2000 & 128 & 128 \\
\hline CricketZ & Motion & 12 & 300 & 390 & 390 & 2000 & 64 & 128 \\
\hline DiatomSizeR & Image & 4 & 345 & 16 & 306 & 2000 & 64 & 64 \\
\hline DisPhOAgeGrp & Image & 3 & 80 & 400 & 139 & 2000 & 128 & 128 \\
\hline DisPhOCorrect & Image & 2 & 80 & 600 & 276 & 2000 & 128 & 128 \\
\hline DisPhTW & Image & 6 & 80 & 400 & 139 & 2000 & 128 & 128 \\
\hline Earthquakes & Sensor & 2 & 512 & 322 & 139 & 2000 & 128 & 128 \\
\hline ECG200 & ECG & 2 & 96 & 100 & 100 & 8000 & 64 & 64 \\
\hline ECG5000 & ECG & 5 & 140 & 500 & 4500 & 2000 & 128 & 128 \\
\hline ECGFiveDays & ECG & 2 & 136 & 23 & 861 & 2000 & 128 & 128 \\
\hline ElectricDevices & Device & $\overline{7}$ & 96 & 8926 & 7711 & 2000 & 128 & 128 \\
\hline FaceAll & Image & 14 & 131 & 560 & 1690 & 2000 & 128 & 128 \\
\hline FaceFour & Image & 4 & 350 & 24 & 88 & 2000 & 128 & 128 \\
\hline FacesUCR & Image & 14 & 131 & 200 & 2050 & 2000 & 128 & 128 \\
\hline FiftyWords & Image & 50 & 270 & 450 & 455 & 2000 & 128 & 128 \\
\hline Fish & Image & 7 & 463 & 175 & 175 & 2000 & 128 & 128 \\
\hline FordA & Sensor & 2 & 500 & 3601 & 1320 & 2000 & 128 & 128 \\
\hline FordB & Sensor & 2 & 500 & 3636 & 810 & 1600 & 128 & 128 \\
\hline GunPoint & Motion & 2 & 150 & 50 & 150 & 2000 & 128 & 128 \\
\hline Ham & Spectro & 2 & 431 & 109 & 105 & 2000 & 128 & 128 \\
\hline HandOutlines & Image & 2 & 2709 & 1000 & 370 & 2000 & 64 & 128 \\
\hline Haptics & Motion & 5 & 1092 & 155 & 308 & 2000 & 128 & 128 \\
\hline Herring & Image & 2 & 512 & 64 & 64 & 2000 & 128 & 128 \\
\hline InlineSkate & Motion & 7 & 1882 & 100 & 550 & 2000 & 128 & 128 \\
\hline InsWingSound & Sensor & 11 & 256 & 220 & 1980 & 1000 & 128 & 128 \\
\hline ItalyPowD & Sensor & 2 & 24 & 67 & 1029 & 2000 & 64 & 128 \\
\hline LargeKApp & Device & 3 & 720 & 375 & 375 & 2000 & 128 & 128 \\
\hline Lightning2 & Sensor & 2 & 637 & 60 & 61 & 4000 & 128 & 128 \\
\hline Lightning7 & Sensor & 7 & 319 & 70 & 73 & 3000 & 32 & 32 \\
\hline Mallat & Simulated & 8 & 1024 & 55 & 2345 & 2500 & 128 & 128 \\
\hline Meat & Spectro & 3 & 448 & 60 & 60 & 2000 & 64 & 128 \\
\hline MedicalImages & Image & 10 & 99 & 381 & 760 & 2000 & 64 & 128 \\
\hline MidPhOAgeGrp & Image & 3 & 80 & 400 & 154 & 2000 & 128 & 128 \\
\hline MidPhOCorrect & Image & 2 & 80 & 600 & 291 & 2000 & 128 & 128 \\
\hline MidPhTW & Image & 6 & 80 & 399 & 154 & 2000 & 128 & 128 \\
\hline MoteStrain & Sensor & 2 & 84 & 20 & 1252 & 2000 & 128 & 128 \\
\hline NonInvECGTh1 & ECG & 42 & 750 & 1800 & 1965 & 2000 & 128 & 128 \\
\hline NonInvECGTh2 & ECG & 42 & 750 & 1800 & 1965 & 2000 & 128 & 128 \\
\hline OliveOil & Spectro & 4 & 570 & 30 & 30 & 6000 & 64 & 128 \\
\hline OSULeaf & Image & 6 & 427 & 200 & 242 & 2000 & 64 & 128 \\
\hline PhalOCorrect & Image & 2 & 80 & 1800 & 858 & 2000 & 64 & 128 \\
\hline Phoneme & Sensor & 39 & 1024 & 214 & 1896 & 2000 & 64 & 128 \\
\hline Plane & Sensor & 7 & 144 & 105 & 105 & 200 & 16 & 16 \\
\hline ProxPhOAgeGrp & Image & 3 & 80 & 400 & 205 & 2000 & 128 & 128 \\
\hline ProxPhOCorrect & Image & 2 & 80 & 600 & 291 & 2000 & 128 & 128 \\
\hline ProxPhTW & Image & 6 & 80 & 400 & 205 & 2000 & 128 & 128 \\
\hline RefDevices & Device & 3 & 720 & 375 & 375 & 2000 & 64 & 64 \\
\hline ScreenType & Device & 3 & 720 & 375 & 375 & 2000 & 64 & 128 \\
\hline ShapeletSim & Simulated & 2 & 500 & 20 & 180 & 2000 & 128 & 128 \\
\hline ShapesAll & Image & 60 & 512 & 600 & 600 & 4000 & 64 & 64 \\
\hline SmlKitApp & Device & 3 & 720 & 375 & 375 & 2000 & 128 & 64 \\
\hline SonyAIBORI & Sensor & 2 & 70 & 20 & 601 & 2000 & 64 & 128 \\
\hline SonyAIBORII & Sensor & 2 & 65 & 27 & 953 & 2000 & 64 & 128 \\
\hline StarLightCurves & Sensor & 3 & 1024 & 1000 & 8236 & 2000 & 64 & 64 \\
\hline Strawberry & Spectro & 2 & 235 & 613 & 370 & 8000 & 64 & 64 \\
\hline SwedishLeaf & Image & 15 & 128 & 500 & 625 & 8000 & 64 & 64 \\
\hline $\begin{array}{l}\text { Symbols } \\
\text { SynControy }\end{array}$ & Image & 6 & 398 & 25 & 995 & 2000 & 64 & 64 \\
\hline SynControl & Simulated & 6 & 60 & 300 & 300 & 4000 & 16 & 128 \\
\hline ToeSegI & Motion & 2 & 277 & 40 & 228 & 2000 & 128 & 64 \\
\hline ToeSegII & Motion & 2 & 343 & 36 & 130 & 2000 & 128 & 32 \\
\hline Trace & Sensor & 4 & 275 & 100 & 100 & 1000 & 64 & 128 \\
\hline TwoLeadECG & ECG & 2 & 82 & 23 & 1139 & 2000 & 64 & 64 \\
\hline TwoPatterns & Simulated & 4 & 128 & 1000 & $\begin{array}{l}4000 \\
3582\end{array}$ & 2000 & 32 & 128 \\
\hline $\begin{array}{l}\text { UWaveAll } \\
\text { UWaY }\end{array}$ & Motion & ${ }_{8}^{8}$ & $\begin{array}{r}945 \\
315\end{array}$ & $\begin{array}{l}896 \\
896\end{array}$ & $\begin{array}{l}3582 \\
3582\end{array}$ & 500 & 16 & 16 \\
\hline UWaveX & Motion & ${ }_{8}^{8}$ & 315 & 896 & 3582 & 2000 & 64 & 16 \\
\hline UWaveY & Motion & ${ }_{8}^{8}$ & 315 & $\begin{array}{l}896 \\
896\end{array}$ & 3582 & 2000 & 64 & 64 \\
\hline UWaveZ & Motion & 8 & 315 & 896 & 3582 & 2000 & 64 & 64 \\
\hline Wafer & Sensor & 2 & 152 & 1000 & 6164 & 1500 & 64 & 64 \\
\hline Wine & Spectro & 25 & 234 & 57 & 54 & 8000 & 64 & 64 \\
\hline WordSynonyms & Image & 25 & 270 & 267 & 638 & 1500 & 64 & 64 \\
\hline Worms & Motion & 5 & 900 & 181 & 77 & 2000 & 64 & 64 \\
\hline WormsTwoClass & $\begin{array}{l}\text { Motion } \\
\text { Image }\end{array}$ & $\frac{2}{2}$ & $\begin{array}{l}900 \\
426\end{array}$ & $\begin{array}{l}181 \\
300\end{array}$ & $\begin{array}{l}77 \\
3000\end{array}$ & $\begin{array}{l}1000 \\
1000\end{array}$ & 16 & 168 \\
\hline & Image & & & & & & 128 & 128 \\
\hline
\end{tabular}


TABLE III. CLASSIFICATION TESTING ERROR AND RANK FOR 85 TIME SERIES DATASETS FROM THE UCR BENCHMARK.

\begin{tabular}{|c|c|c|c|c|c|c|c|c|}
\hline Dataset & & & & & lassificati & Method & ind Testi & Error \\
\hline & $G R U-F C N$ & $F C N$ & LSTMFCN & ALSTMFCN & ResNet & MCNN & $M L P$ & COTE \\
\hline Adiac & 0.127 & 0.143 & 0.141 & 0.139 & 0.174 & 0.231 & 0.248 & 0.233 \\
\hline ArrowHead & 0.085 & 0.120 & 0.102 & 0.119 & 0.183 & 1 & 0.292 & 0.138 \\
\hline Beef & 0.100 & 0.250 & 0.167 & 0.233 & 0.233 & 0.367 & 0.167 & 0.133 \\
\hline BeetleFly & 0.050 & 0.050 & 0.050 & 0.050 & 0.200 & l & 0.200 & 0.050 \\
\hline BirdChicken & $\mathbf{0}$ & 0.050 & $\mathbf{0}$ & $\mathbf{0}$ & 0.100 & I & 0.400 & 0.150 \\
\hline Car & 0.016 & 0.050 & 0.033 & 0.159 & 0.067 & l & 0.117 & I \\
\hline $\mathrm{CBF}$ & 0 & 0.008 & 0.003 & 0.004 & 0.006 & 0.002 & 0.14 & 0.001 \\
\hline ChloConc & 0.002 & 0.157 & 0.191 & 0.193 & 0.172 & 0.203 & 0.125 & 0.314 \\
\hline CinCECGTorso & 0.124 & 0.187 & 0.191 & 0.193 & 0.172 & 0.058 & 0.158 & 0.064 \\
\hline Coffee & $\mathbf{0}$ & $\mathbf{0}$ & $\mathbf{0}$ & $\mathbf{0}$ & $\mathbf{0}$ & 0.036 & $\mathbf{0}$ & $\mathbf{0}$ \\
\hline Computers & 0.148 & 0.152 & 0.136 & 0.123 & 0.176 & I & 0.504 & 0.240 \\
\hline CricketX & 0.156 & 0.185 & 0.193 & 0.203 & 0.179 & 0.182 & 0.431 & 0.154 \\
\hline CricketY & 0.156 & 0.208 & 0.183 & 0.185 & 0.195 & 0.154 & 0.405 & 0.167 \\
\hline Cricketz & 0.154 & 0.187 & 0.190 & 0.175 & 0.169 & 0.142 & 0.408 & 0.128 \\
\hline DiatomSizeR & 0.036 & 0.069 & 0.046 & 0.063 & 0.069 & 0.023 & 0.036 & 0.082 \\
\hline DisPhOAgeGr & 0.142 & 0.165 & 0.145 & 0.137 & 0.202 & I & 0.178 & 0.229 \\
\hline DisPhOCorrect & 0.168 & 0.188 & 0.168 & 0.163 & 0.180 & I & 0.195 & 0.238 \\
\hline DisPhalanxTW & 0.180 & 0.210 & 0.185 & 0.185 & 0.260 & l & 0.375 & 0.317 \\
\hline Earthquakes & 0.171 & 0.199 & 0.177 & 0.173 & 0.214 & l & 10.208 & 1 \\
\hline ECG200 & 0.080 & 0.100 & 0.100 & 0.090 & 0.130 & i & 0.210 & 0.150 \\
\hline ECG5000 & 0.052 & 0.059 & 0.053 & 0.052 & 0.069 & i & 0.068 & 0.054 \\
\hline ECG5Days & $\mathbf{0}$ & 0.010 & 0.011 & 0.009 & 0.045 & $\mathbf{0}$ & 0.030 & $\mathbf{0}$ \\
\hline ElectricDevices & 0.037 & 0.277 & 0.037 & 0.037 & 0.272 & I & 0.360 & 0.230 \\
\hline FaceAll & 0.040 & 0.071 & 0.060 & 0.045 & 0.166 & 0.235 & 0.115 & 0.105 \\
\hline FaceFour & 0.136 & 0.068 & 0.057 & 0.057 & 0.068 & 0 & 0.167 & 0.091 \\
\hline FourUCR & 0.050 & 0.052 & 0.071 & 0.057 & 0.042 & 0.063 & 0.185 & 0.057 \\
\hline FiftyWords & 0.167 & 0.321 & 0.196 & 0.176 & 0.273 & 0.190 & 0.288 & 0.191 \\
\hline Fish & 0.006 & 0.029 & 0.017 & 0.023 & 0.011 & 0.051 & 0.126 & 0.029 \\
\hline FordA & 0.074 & 0.094 & 0.072 & 0.073 & 0.072 & I & 0.231 & I \\
\hline FordB & 0.083 & 0.117 & 0.088 & 0.081 & 0.100 & I & 0.371 & 1 \\
\hline GunPoint & 0 & 0 & 0 & 0 & 0.007 & 0 & 0.067 & 0.007 \\
\hline Ham & 0.209 & 0.238 & 0.209 & 0.228 & 0.219 & I & 0.162 & 0.334 \\
\hline HandOutlines & 0.112 & 0.224 & 0.113 & 0.358 & 0.139 & i & 0.117 & 0.068 \\
\hline Haptics & 0.455 & 0.449 & 0.425 & 0.435 & 0.495 & 0.530 & 0.539 & 0.488 \\
\hline Herring & 0.250 & 0.297 & 0.250 & 0.265 & 0.406 & I & 0.360 & 0.313 \\
\hline InlineSkate & 0.625 & 0.589 & 0.534 & 0.507 & 0.635 & 0.618 & 0.649 & 0.551 \\
\hline InsWSound & 0.446 & 0.598 & 0.342 & 0.329 & 0.469 & 1 & 0.369 & 1 \\
\hline ItalyPower & 0.027 & 0.030 & 0.037 & 0.040 & 0.040 & 0.030 & 0.034 & 0.036 \\
\hline LKitApp & 0.090 & 0.104 & 0.090 & 0.083 & 0.107 & j & 0.520 & 0.136 \\
\hline Lightening2 & 0.197 & 0.197 & 0.197 & 0.213 & 0.246 & 0.164 & 0.279 & 0.164 \\
\hline Lightening7 & 0.137 & 0.137 & 0.164 & 0.178 & 0.164 & 0.219 & 0.356 & 0.247 \\
\hline MÁLLAT & 0.048 & 0.020 & 0.019 & 0.016 & 0.021 & 0.057 & 0.064 & 0.036 \\
\hline Meat & 0.066 & 0.033 & 0.116 & 0.033 & $\mathbf{0}$ & 1 & 0 & 0.067 \\
\hline MedicalImages & 0.199 & 0.208 & 0.199 & 0.204 & 0.228 & 0.260 & 0.271 & 0.258 \\
\hline MidPhOAgeGrp & 0.187 & 0.232 & 0.188 & 0.189 & 0.240 & 1 & 0.193 & 0.169 \\
\hline MidPhOCorrect & 0.160 & 0.205 & 0.160 & 0.163 & 0.207 & I & 0.442 & 0.403 \\
\hline MidPhTW & 0.363 & 0.388 & 0.383 & 0.373 & 0.393 & I & 0.429 & 0.429 \\
\hline MoteStrain & 0.076 & 0.050 & 0.061 & 0.064 & 0.105 & 0.079 & 0.131 & 0.085 \\
\hline NonInvECGTh1 & 0.034 & 0.039 & 0.035 & 0.025 & 0.052 & 0.064 & 0.058 & 0.093 \\
\hline NonInvECGTh2 & 0.035 & 0.045 & 0.038 & 0.034 & 0.049 & 0.060 & 0.057 & 0.073 \\
\hline OliveOil & 0.012 & 0.167 & 0.133 & 0.067 & 0.133 & 0.133 & 0.600 & 0.100 \\
\hline OSULeaf & $\mathbf{0}$ & 0.012 & 0.004 & 0.004 & 0.021 & 0.271 & 0.430 & 0.145 \\
\hline PhalOCorrect & 0.165 & 0.174 & 0.177 & 0.170 & 0.175 & I & 0.164 & 0.194 \\
\hline Phoneme & 0.644 & 0.655 & 0.650 & 0.640 & 0.676 & I & 0.902 & I \\
\hline Plane & $\mathbf{0}$ & $\mathbf{0}$ & $\mathbf{0}$ & $\mathbf{0}$ & $\mathbf{0}$ & I & 0.019 & 1 \\
\hline ProxPhOeAgeGrp & 0.117 & 0.151 & 0.117 & 0.107 & 0.151 & I & 0.135 & 0.121 \\
\hline ProxPhOCorrect & 0.079 & 0.100 & 0.065 & 0.075 & 0.082 & i & 0.200 & 0.142 \\
\hline ProxPhTW & 0.167 & 0.190 & 0.167 & 0.173 & 0.193 & I & 0.210 & 0.186 \\
\hline RefDevices & 0.407 & 0.467 & 0.421 & 0.429 & 0.472 & I & 0.632 & 0.443 \\
\hline ScreenType & 0.297 & 0.333 & 0.351 & 0.341 & 0.293 & I & 0.614 & 0.411 \\
\hline ShapeletSim & 0.011 & 0.133 & 0.011 & 0.011 & $\mathbf{0}$ & l & 0.528 & $\mathbf{0}$ \\
\hline ShapesAll & 0.097 & 0.102 & 0.098 & 0.100 & 0.088 & I & 0.350 & 0.095 \\
\hline SmlKitApp & 0.186 & 0.197 & 0.184 & 0.203 & 0.203 & I & 0.667 & 0.147 \\
\hline SonyAIBORI & 0.017 & 0.032 & 0.018 & 0.030 & 0.015 & 0.230 & 0.273 & 0.146 \\
\hline SonyAIBORII & 0.018 & 0.038 & 0.022 & 0.025 & 0.038 & 0.070 & 0.161 & 0.076 \\
\hline StarLightCurves & 0.025 & 0.033 & 0.024 & 0.023 & 0.029 & 0.023 & 0.043 & 0.031 \\
\hline Strawberry & 0.013 & 0.031 & 0.013 & 0.013 & 0.042 & 1 & 0.038 & 0.030 \\
\hline SwedishLeaf & 0.016 & 0.034 & 0.021 & 0.014 & 0.042 & 0.066 & 0.107 & 0.046 \\
\hline Symbols & 0.024 & 0.038 & 0.016 & 0.013 & 0.128 & 0.049 & 0.147 & 0.046 \\
\hline SynControl & $\mathbf{0}$ & 0.010 & 0.003 & 0.006 & $\mathbf{0}$ & 0.003 & 0.050 & $\mathbf{0}$ \\
\hline ToeSeg 1 & 0.021 & 0.031 & 0.013 & 0.013 & 0.035 & I & 0.500 & 0.018 \\
\hline ToeSeg2 & 0.076 & 0.085 & 0.084 & 0.077 & 0.138 & I & 0.408 & 0.047 \\
\hline Trace & $\mathbf{0}$ & $\mathbf{0}$ & $\mathbf{0}$ & 0 & $\mathbf{0}$ & 0 & 0.180 & 0.010 \\
\hline TwoLeadECG & $\mathbf{0}$ & $\mathbf{0}$ & 0.001 & 0.001 & 0 & 0.001 & 0.147 & 0.015 \\
\hline TwoPatterns & 0.009 & 0.103 & 0.003 & 0.003 & 0 & 0.002 & 0.114 & 0 \\
\hline UWaveAll & 0.078 & 0.174 & 0.096 & 0.107 & 0.132 & 1 & 0.253 & 0.161 \\
\hline UWaveX & 0.171 & 0.246 & 0.151 & 0.152 & 0.213 & 0.180 & 0.232 & 0.196 \\
\hline UWaveY & 0.240 & 0.275 & 0.233 & 0.234 & 0.332 & 0.268 & 0.297 & 0.267 \\
\hline UWaveZ & 0.237 & 0.271 & 0.203 & 0.202 & 0.245 & 0.232 & 0.295 & 0.265 \\
\hline Wafer & 0.001 & 0.003 & 0.001 & 0.002 & 0.003 & 0.002 & 0.004 & 0.001 \\
\hline Wine & 0.111 & 0.111 & 0.111 & 0.111 & 0.204 & l & 0.056 & 0.223 \\
\hline WordSynonyms & 0.262 & 0.420 & 0.329 & 0.332 & 0.368 & 0.276 & 0.406 & 0.266 \\
\hline Worms & 0.325 & 0.331 & 0.325 & 0.320 & 0.381 & I & 0.585 & 0.442 \\
\hline WormsTwoClass & 0.209 & 0.271 & 0.226 & 0.198 & 0.265 & I & 0.403 & 0.221 \\
\hline Yoga & 0.090 & 0.098 & 0.082 & 0.081 & 0.142 & 0.112 & 0.145 & 0.113 \\
\hline no. best & 39 & 9 & 19 & 25 & 13 & 5 & 3 & 11 \\
\hline Arith AVG Rank & 2.947 & 5.841 & 3.818 & 3.729 & 6.035 & 9.118 & 9.100 & 6.071 \\
\hline MPCE & 0.0308 & 0.0387 & 0.0327 & 0.0342 & 0.0415 & 0.1853 & 0.0725 & 0.0629 \\
\hline
\end{tabular}


Each dataset is divided into training and testing sets. The number of classes in each time series, the length and the size of both the training and test sets are shown in Table II based on the datasets description in [14]. The UCR benchmark datasets have different types of collected sources: 29 datasets of image source, 6 spectro source, 5 simulated source, 19 sensor source, 6 device source, 12 motion source, and 6 electrocardiogram (ECG) source. In addition, as we mentioned in the previous Section, Table II also shows the number of epochs through training, and the batch sizes of the training and testing stages based on our experiments.

We compared our GRU-FCN with several state-of-the-art time series methods that also were studied in [4] and [5]. These included FCN [4] which is based on a fully convolutional network, LSTM-FCN [5], ALSTM-FCN [5], that are based on long short-term memory and fully convolutional networks, ResNet [4] which based on convolutional residual networks, multi-scale convolution neural networks model (MCNN) [13], multi-layered perceptrons model (MLP) [4], collective of transformation-based ensembles model (COTE) [12] which based on transformation ensembles, dynamic time warping model (DTW) [30] that is based on a weighted dynamic time warping mechanism, PROP model [11] which is based on elastic distance measures, BOSS model [9] that based on noise reduction in the time series representation, time series based on a bag-of-features representation (TSBF) model [10], and Euclidean distance (ED) model [14]. Our model shows the overall highest number of being the best classifier for 39 time series out of 85 . Our model also shows the overall smallest classification error, arithmetic average rank, and mean per-class classification error (MPCE) compared to the other models as shown in Table III.

Table IV shows a comparison between the number of parameters, training time and memory required to save the trainable weights of the GRU-FCN and both LSTM-FCN and ALSTM-FCN models as the existing LSTM-based to-date univariate classification models over the UCR 85 datasets. The GRU-FCN has a smaller number of parameters for all the datasets. The GRU-FCN saves overall $1207 \mathrm{~KB}$, and $5719 \mathrm{~KB}$ memory requirements to save the trained model's weight; and 106.065, and 62.271 minutes to train the models over the UCR datasets comparing to the LSTM-FCN and ALSTM-FCN, respectively. Therefore, the GRU-FCN is preferable as a low budget classification model with high accuracy performance.

We evaluated our model using the Mean Per-Class Error (MPCE) used in [4] to evaluate the performance of a classification method over multiple datasets. The MPCE for a given model is calculated based on the per-class error (PCE) as follows:

$$
\begin{aligned}
\mathrm{PCE}_{m} & =\frac{e_{m}}{c_{m}} \\
\mathrm{MPCE} & =\frac{1}{M} \sum_{m=1}^{M} \mathrm{PCE}_{m}
\end{aligned}
$$

where $e_{m}$ is the error rate for dataset $m$ consisting of $c_{m}$ classes. $M$ is the number of tested datasets.

Table III shows the MPCE value for our GRU-FCN and other state-of-the-art models on the UCR benchmark datasets [14]. The results obtained by implementing GRU-FCN and generating LSTM-FCN, and ALSTM models based on their actual implementation on Github. For the other models, we obtained the results from their own publications. Our GRUFCN has the smallest MPCE value compared to the other stateof-the-art classification models. This means that generally, our GRU-FCN model performance across the different datasets is higher than the other state-of-the-art models.

Fig. 4, 5, 6, 7 are showing the loss value of both the training and validation processed of datasets. Each of these figures represents the loss process over image, motion, simulated, and source-obtained datasets from the UCR benchmark datasets respectively. These figures show that the average difference between the training and validation loss for the GRU-FCN is smaller than the LSTM-FCN and ALSTM-FCN models.

Table $\mathrm{V}$ shows the f1-score (also known as F-score or Fmeasure) [31], [32] for GRU-FCN, LSTM-FCN, and ALSTMFCN classifiers. The f1-score shows the overall measure of a model's accuracy over each dataset used. The f1-score measuring based on both the precision and recall values of the classification model [31], [32]. The f1-score is calculated as follows [31], [32]:

$$
\begin{aligned}
\text { precision } & =\frac{T P}{T P+F P} \\
\text { recall } & =\frac{T P}{T P+F N} \\
f 1-\text { score } & =2 \times \frac{\text { precision } \times \text { recall }}{\text { precision }+ \text { recall }}
\end{aligned}
$$

where TP, FP, FN stands for true-positive, false-positive and false-negative respectively. The GRU-FCN shows the highest f1-score for 53 out of 85 datasets comparing to the LSTMFCN and ALSTM-FCN that both of these models have the highest f1-score for only 29 out of 85 datasets.

Fig. 8 shows the critical difference diagram [33] for $\mathrm{Ne}$ menyi or Bonferroni-Dunn test [34] with $\alpha=0.05$ on our GRU-FCN and the state-of-the-art models based on the ranks arithmetic mean on the UCR benchmark datasets. This graph shows the significant classification accuracy improvement of our GRU-FCN compared to the other state-of-the-art models.

The Wilcoxon signed-rank test is one of the substantial tests to provide the classification method efficiency [35], [36]. Table VI shows the Wilcoxon signed-rank test [35], [37] among the twelve state-of-the-art classification models. This provides the overall accuracy evidence of each of the twelve classification methods.

\section{Conclusion}

The proposed GRU-FCN classification model shows that replacing the LSTM by a GRU enhances the classification accuracy without requiring extra algorithm enhancements such as fine-tuning or attention algorithms. This The GRU also has a smaller architecture that requires fewer computations than the LSTM. Moreover, the GRU-based model requires a smaller number of trainable parameters, memory, and training time compared to the LSTM-based models. Furthermore, the proposed GRU-FCN classification model achieves the performance of state-of-the-art models and has the highest 
TABLE IV. A COMPARISON BETWEEN THE GRU-FCN AND LSTM-BASED CLASSIFICATION MODELS FOR THE NUMBER OF PARAMETERS, TRAINING TIME (MINUTES), AND MEMORY (KB) REQUIRED TO SAVE THE MODEL WEIGHTS ON THE UCR 85 DATASETS [14].

\begin{tabular}{|c|c|c|c|c|c|c|c|c|c|}
\hline Dataset & & Imber of Para & eters & Trai & ng Time (Min & & & Memory (KB & \\
\hline & GRU-FCN & LSTM-FCN & ALSTM-FCN & $G R U-F C N$ & LSTM-FCN & ALSTM & $G R U-F C N$ & LSTM-FCN & LSTM-FCN \\
\hline Adiac & 275,237 & 276,717 & 283,837 & 9.597 & 9.560 & 10.056 & 1,114 & 1,119 & 1,150 \\
\hline ArrowHead & 272,379 & 274,459 & 284,579 & 4.134 & 4.303 & 4.692 & 1,103 & 1,111 & 1,151 \\
\hline Beef & 277,909 & 281,741 & 300,621 & 3.896 & 4.804 & 4.889 & 1,124 & 1,139 & 1,215 \\
\hline BeetleFly & 278,506 & 282,674 & 303,234 & 3.937 & 4.208 & 4.545 & 1,126 & 1,144 & 1,225 \\
\hline BirdChicken & 278,506 & 282,674 & 303,234 & 3.437 & 3.760 & 4.131 & 1,126 & 1,144 & 1,225 \\
\hline Car & 280,340 & 285,028 & 308,188 & 1.899 & 1.972 & 2.045 & 1,134 & 1,152 & 1.245 \\
\hline $\mathrm{CBF}$ & 269,427 & 270,523 & 275,723 & 5.243 & 5.248 & 5.339 & 1,092 & 1,096 & 1,117 \\
\hline ChloConc & 270,339 & 271,739 & 278,459 & 13.324 & 14.601 & 14.813 & 1,095 & 1,110 & 1,127 \\
\hline CinCECGTorso & 305,828 & 319,012 & 384,652 & 6.087 & 6.594 & 7.003 & 1,233 & 1,285 & 1,544 \\
\hline Coffee & 273,082 & 275,442 & 286,962 & 0.504 & 0.524 & 0.540 & 1,104 & 1,115 & 1,161 \\
\hline Computers & 283,498 & 289,330 & 318,210 & 7.722 & 8.049 & 8.436 & 1,145 & 1,170 & 1,283 \\
\hline CricketX & 274,788 & 277,260 & 289,340 & 6.850 & 7.124 & 7.292 & 1,112 & 1,122 & 1,171 \\
\hline CricketY & 274,788 & 277,260 & 289,340 & 6.673 & 6.978 & 7.224 & 1,112 & 1,122 & 1,171 \\
\hline Cricketz & 274,788 & 277,260 & 289,340 & 8.601 & 8.933 & 9.539 & 1,112 & 1,122 & 1,171 \\
\hline DiatomSizeR & 274,772 & 277,604 & 291,484 & 2.886 & 3.016 & 3.066 & 1,112 & 1,123 & 1,180 \\
\hline DisPhOAgeGrp & 268,275 & 268,987 & 272,267 & 2.346 & 2.439 & 5.056 & 1,087 & 1,090 & 1,103 \\
\hline DisPhOCorrect & 268,138 & 268,850 & 272,130 & 3.554 & 3.791 & 3.980 & 1,085 & 1,090 & 1,103 \\
\hline DisPhTW & 268,686 & 269,398 & 272,678 & 2.611 & 2.723 & 2.876 & 1,088 & $\begin{array}{l}1,091 \\
1,091\end{array}$ & 1,106 \\
\hline Earthquakes & 278,506 & 282,674 & 303,234 & 4.998 & 5.507 & 5.547 & 1,126 & 1,144 & 1,225 \\
\hline ECG200 & 268,522 & 269,362 & 273,282 & 5.305 & 5.599 & 6.125 & 1,087 & 1,092 & 1,108 \\
\hline ECG5000 & 269,989 & 271,181 & 276,861 & 13.223 & 13.797 & 14.162 & 1,093 & 1,098 & 1,123 \\
\hline ECG5Days & 269,482 & 270,642 & 276,162 & 2.433 & 2.481 & 2.494 & 1,090 & 1,097 & 1,119 \\
\hline ElectricDevices & $\begin{array}{l}209,402 \\
269,207\end{array}$ & $\begin{array}{l}270,042 \\
270,047\end{array}$ & $\begin{array}{l}270,102 \\
273,967\end{array}$ & 67.350 & $\begin{array}{l}75.401 \\
75.44\end{array}$ & 65.879 & 1,090 & $\begin{array}{l}1,091 \\
1,093\end{array}$ & 1,111 \\
\hline FaceAll & 271,006 & 272,126 & 277,446 & 7.465 & 7.753 & 7.812 & 1,097 & 1,101 & 1,125 \\
\hline FaceFour & 274,892 & 277,764 & 291,844 & 1.072 & 1.101 & 1.197 & 1,112 & 1,123 & 1,181 \\
\hline FourUCR & 271,006 & 272,126 & 277,446 & 7.609 & 7.722 & 8.241 & 1,097 & 1,101 & 1,125 \\
\hline FiftyWords & 279,274 & 281,506 & 292,386 & 6.052 & 6.353 & $\begin{array}{l}.241 \\
6.428\end{array}$ & 1,129 & $\begin{array}{l}1,101 \\
1,138\end{array}$ & 1,183 \\
\hline Fish & 278,015 & 281,791 & 300,391 & 3.770 & 3.850 & 3.912 & 1,125 & 1,139 & 1,214 \\
\hline FordA & 278,218 & 282,290 & 302,370 & 43.135 & 44.861 & 47.525 & 1,124 & 1,142 & 1,221 \\
\hline FordB & 278,218 & 282,290 & 302,370 & 26.781 & 27.341 & 27.890 & 1,124 & 1,142 & 1,221 \\
\hline GunPoint & 269,818 & 271,090 & 277,170 & 1.003 & 1.046 & 1.138 & 1,092 & 1,098 & 1,123 \\
\hline Ham & $\begin{array}{l}207,010 \\
276,562\end{array}$ & 280,082 & 297,402 & 2.048 & 2.127 & 2.160 & 1,118 & $\begin{array}{l}1,090 \\
1,133\end{array}$ & 1,202 \\
\hline HandOutlines & 331,234 & 352,978 & 461,418 & 61.902 & 62.375 & 63.393 & 1,332 & 1,418 & 1,842 \\
\hline Haptics & 292,837 & 301,645 & 345,405 & 9.787 & 10.023 & 10.631 & 1,183 & 1,217 & 1,390 \\
\hline Herring & 278,506 & 282,674 & 303,234 & 1.633 & 1.668 & 1.706 & 1,126 & 1,144 & 1,225 \\
\hline InlineSkate & 312,071 & 327,199 & 402,559 & 16.439 & 16.772 & 17.853 & 1,258 & 1,317 & 1,614 \\
\hline InsWingSound & 273,595 & 275,715 & 286,035 & 4.332 & 4.510 & 4.599 & 1,107 & 1,115 & 1,158 \\
\hline $\begin{array}{l}\text { ItalyPowD } \\
\text { lation }\end{array}$ & 266,794 & 267,058 & 268,098 & 2.719 & 3.015 & 3.048 & 1,080 & 1,083 & 1,087 \\
\hline LargeKApp & 283,635 & 289,467 & 318,347 & 10.786 & 12.008 & 11.640 & 1,147 & 1,170 & 1,283 \\
\hline Lightening2 & 281,506 & 286,674 & 312,234 & 3.887 & 3.940 & 4.065 & 1,137 & 1,159 & 1,260 \\
\hline Lightening7 & $\begin{array}{l}201,550 \\
274,559\end{array}$ & $\begin{array}{l}200,183 \\
277,183\end{array}$ & 290,023 & $\begin{array}{l}3.001 \\
4.091\end{array}$ & 4.811 & 4.477 & 1,111 & 1,121 & 1,174 \\
\hline MALLAT & 291,616 & 299,880 & 340,920 & 34.911 & 37.448 & 38.080 & 1,178 & 1,210 & 1,373 \\
\hline Meat & 277,107 & 280,763 & 298,763 & 1.698 & 1.737 & 1.832 & 1,122 & 1,136 & 1,207 \\
\hline MedicalImages & 269,690 & 270,554 & 274,594 & 5.361 & 5.456 & 6.498 & 1,092 & 1,095 & 1,114 \\
\hline MidPhOAgeGrp & 268,275 & 268,987 & 272,267 & 1.802 & 2.138 & 2.182 & 1,087 & 1,090 & 1,103 \\
\hline MidPhOCorrect & 268,138 & 268,850 & 272,130 & 3.219 & 3.374 & 3.528 & 1,085 & 1,090 & 1,103 \\
\hline MidPhTW & 268,686 & 269,398 & 272,678 & 2.271 & 2.340 & 2.321 & 1,088 & 1,091 & 1,106 \\
\hline MoteStrain & 268,234 & 268,978 & 272,418 & 2.398 & 2.423 & 2.481 & 1,085 & 1,090 & 1,104 \\
\hline NonInvECGTh1 & 289,698 & 295,770 & 325,850 & 61.809 & 61.853 & 71.308 & 1,170 & 1,194 & 1,314 \\
\hline NonInvECGTh2 & $\begin{array}{l}289,090 \\
289,698\end{array}$ & 295,770 & 325,850 & $\begin{array}{l}50.012 \\
59.212\end{array}$ & $\begin{array}{l}01.053 \\
60.554\end{array}$ & 60.754 & 1,170 & $\begin{array}{l}1,194 \\
1,194\end{array}$ & 1,314 \\
\hline OliveOil & 280,172 & 284,804 & 307,684 & 3.267 & 3.670 & 4.073 & 1,133 & 1,151 & 1,243 \\
\hline OSULeaf & 277,014 & 280,502 & 297,662 & 4.962 & 5.096 & 5.409 & 1,121 & 1,134 & 1,204 \\
\hline PhalOCorrect & 268,138 & 268,850 & 272,130 & 16.319 & 19.269 & 21.159 & 1,085 & 1,090 & 1,103 \\
\hline Phoneme & 295,863 & 304,127 & 345,167 & 29.778 & 31.34 & 37.147 & 1,194 & 1,226 & 1,389 \\
\hline Plane & 270,359 & 271,583 & 277,423 & 0.497 & 0.502 & 0.575 & 1,095 & 1,099 & 1,125 \\
\hline ProxPhOAgeGrp & 268,275 & 268,987 & 272,267 & 3.550 & 3.601 & 3.605 & 1,087 & 1,090 & 1,103 \\
\hline ProxPhOCorrect & 268,138 & 268,850 & 272,130 & 4.142 & 4.538 & 4.678 & 1,085 & 1,090 & 1,103 \\
\hline ProxPhTW & 268,686 & 269,398 & 272,678 & 2.050 & 2.201 & 2.126 & 1,088 & 1,091 & 1,106 \\
\hline RefDevices & 283,635 & 289,467 & 318,347 & 12.878 & 14.160 & 14.460 & 1,147 & $\begin{array}{l}1,170 \\
1,170\end{array}$ & 1,283 \\
\hline ScreenType & 283,635 & 289,467 & 318,347 & 13.327 & 13.890 & 14.283 & 1,147 & 1,170 & 1,283 \\
\hline ShapeletSim & 278,218 & $\begin{array}{l}28,, 401 \\
282,290\end{array}$ & 302,370 & 1.596 & $\begin{array}{l}1.628 \\
1.628\end{array}$ & 2.004 & 1,124 & 1,142 & $\begin{array}{l}1,221 \\
1,221\end{array}$ \\
\hline ShapesAll & 286,452 & 290,620 & 311,180 & 34.243 & 36.523 & 37.627 & 1,157 & 1,173 & 1,256 \\
\hline SmlKitApp & 283,635 & 289,467 & 318,347 & 12.417 & 12.92 & 14.248 & 1,147 & 1,170 & 1,283 \\
\hline SonyAIBORI & 267,898 & $\begin{array}{l}209,401 \\
268,530\end{array}$ & $\begin{array}{l}510,341 \\
271,410\end{array}$ & 0.982 & 1.931 & $\begin{array}{r}14.040 \\
2.042\end{array}$ & $\begin{array}{l}1,147 \\
1,084\end{array}$ & 1,088 & $\begin{array}{l}1,203 \\
1,100\end{array}$ \\
\hline SonyAIBORII & 267,778 & 268,370 & 271,050 & 2.492 & 2.496 & 2.873 & 1,084 & 1,088 & 1,099 \\
\hline StarLightCurves & 290,931 & $\begin{array}{l}200,195 \\
299,195\end{array}$ & 340,235 & 151.538 & $\begin{array}{l}157.143 \\
157\end{array}$ & 161.447 & $\begin{array}{l}1,004 \\
1,176\end{array}$ & $\begin{array}{l}1,000 \\
1,208\end{array}$ & $\begin{array}{l}1,099 \\
1,369\end{array}$ \\
\hline Strawberry & 271,858 & 273,810 & 283,290 & 39.138 & 40.408 & $\begin{array}{r}42.769 \\
\end{array}$ & 1,100 & 1,109 & 1,147 \\
\hline SwedishLeaf & 271,071 & 272,167 & 277,367 & $\begin{array}{r}6.931 \\
6.930\end{array}$ & $\begin{array}{r}r .400 \\
7.572\end{array}$ & 7.891 & 1,098 & 1,102 & 1,125 \\
\hline Symbols & 276,318 & 279,574 & 295,574 & 6.176 & 6.543 & 6.736 & 1,118 & 1,131 & 1,196 \\
\hline SynControl & 268,206 & 268,758 & 271,238 & 20.562 & $\begin{array}{l}0.043 \\
21.735\end{array}$ & $\begin{array}{l}0.1709 \\
23.209\end{array}$ & 1,086 & $\begin{array}{l}1,131 \\
1,088\end{array}$ & 1,101 \\
\hline ToeSeg1 & 272,866 & 275,154 & 286,314 & 1.824 & 1.846 & 1.900 & 1,104 & 1,114 & 1,158 \\
\hline ToeSeg2 & 274,450 & 277,266 & 291,066 & 1.415 & 1.549 & 1.629 & 1,110 & 1,122 & 1,177 \\
\hline Trace & 273,092 & $\begin{array}{l}211,200 \\
275,364\end{array}$ & 286,444 & $\begin{array}{l}1.415 \\
0.977\end{array}$ & 1.021 & 1.093 & 1,105 & 1,114 & 1,160 \\
\hline TwoLeadECG & 268,186 & 268,914 & 272,274 & 3.053 & 3.535 & 3.498 & 1,085 & 1,090 & 1,104 \\
\hline TwoPatterns & 269,564 & 270,660 & 275,860 & 33.994 & 37.673 & 38.303 & 1,092 & 1,096 & 1,119 \\
\hline UWaveAll & 289,720 & 297,352 & 335,232 & 24.983 & 28.702 & 28.874 & 1,170 & 1,200 & 1,351 \\
\hline UWaveX & 274,600 & 277,192 & 289,872 & 30.214 & 32.095 & 33.573 & 1,111 & 1,121 & 1,173 \\
\hline UWaveY & 274,600 & 277,192 & 289,872 & 30.214 & 31.526 & 32.526 & 1,111 & 1,121 & 1,173 \\
\hline UWaveZ & 274,600 & 277,192 & 289,872 & 30.214 & 31.881 & 33.573 & 1,111 & 1,121 & 1,173 \\
\hline Wafer & 269,866 & 271,154 & 277,314 & 20.438 & 21.835 & 22.018 & 1,092 & 1,099 & 1,123 \\
\hline Wine & 271,834 & 273,778 & 283,218 & 3.771 & 4.021 & 4.530 & 1,099 & 1,109 & 1,146 \\
\hline WordSynonyms & 275,849 & 278,081 & 288,961 & 4.911 & 5.155 & 5.498 & 1,116 & 1,125 & 1,170 \\
\hline Worms & 288,229 & 295,501 & 331,581 & 4.484 & 4.669 & 5.019 & 1,165 & 1,193 & 1,336 \\
\hline WormsTwoClass & 287,818 & 295,090 & 331,170 & 3.536 & 3.586 & 4.134 & 1,162 & 1,192 & 1,334 \\
\hline Yoga & 276,442 & 279,922 & 297,042 & 10.970 & 11.606 & 10.753 & 1,118 & 1,133 & 1,200 \\
\hline Total & $23,555,876$ & $23,849,100$ & $25,291,420$ & 1145.645 & 1207.916 & 1251.71 & 95,273 & 96,480 & 100,992 \\
\hline
\end{tabular}


TABLE V. THE F1-SCORE VALUE OF THE PROPOSED GRU-FCN MODEL AND THE LSTM-BASED ARCHITECTURES OVER THE UCR BENCHMARK DATASETS [14].

\begin{tabular}{|c|c|c|c|}
\hline \multirow[t]{2}{*}{ Dataset } & \multicolumn{3}{|c|}{ f1-Score } \\
\hline & $G R U-F C N$ & LSTM-FCN & ALSTM-FCN \\
\hline Adiac & 0.795 & 0.770 & 0.780 \\
\hline ArrowHead & 0.711 & 0.694 & 0.695 \\
\hline Beef & 0.819 & 0.873 & 0.765 \\
\hline BeetleFly & 1.0 & 1.0 & 0.949 \\
\hline BirdChicken & 1.0 & 1.0 & 1.0 \\
\hline Car & 0.954 & 0.952 & 0.947 \\
\hline $\mathrm{CBF}$ & 0.995 & 0.994 & 0.989 \\
\hline ChlorineCon & 0.766 & 0.791 & 0.767 \\
\hline CinCECGTorso & 0.379 & 0.321 & 0.375 \\
\hline Coffee & 1.0 & 1.0 & 1.0 \\
\hline Computers & 0.916 & 0.914 & 0.913 \\
\hline CricketX & 0.786 & 0.782 & 0.784 \\
\hline CricketY & 0.756 & 0.786 & 0.776 \\
\hline CricketZ & 0.779 & 0.778 & 0.761 \\
\hline DiatomSizeR & 0.926 & 0.926 & 0.935 \\
\hline DisPhOAgeGrp & 0.645 & 0.614 & 0.636 \\
\hline DisPhOCorrect & 0.813 & 0.804 & 0.813 \\
\hline DisPhTW & 0.477 & 0.469 & 0.479 \\
\hline Earthquakes & 0.483 & 0.466 & 0.466 \\
\hline ECG200 & 0.910 & 0.900 & 0.909 \\
\hline ECG5000 & 0.253 & 0.251 & 0.263 \\
\hline ECGFiveDays & 0.991 & 0.991 & 0.991 \\
\hline ElectricDevices & 0.195 & 0.196 & 0.197 \\
\hline FaceAll & 0.137 & 0.134 & 0.136 \\
\hline FaceFour & 0.960 & 0.949 & 0.949 \\
\hline FacesUCR & 0.892 & 0.898 & 0.896 \\
\hline 50words & 0.353 & 0.330 & 0.353 \\
\hline Fish & 0.962 & 0.964 & 0.957 \\
\hline FordA & 0.926 & 0.928 & 0.928 \\
\hline FordB & 0.928 & 0.930 & 0.929 \\
\hline GunPoint & 1.0 & 1.0 & 1.0 \\
\hline Ham & 0.788 & 0.788 & 0.770 \\
\hline HandOutlines & 0.875 & 0.873 & 0.866 \\
\hline Haptics & 0.528 & 0.523 & 0.515 \\
\hline Herring & 0.717 & 0.722 & 0.694 \\
\hline InlineSkate & 0.454 & 0.474 & 0.446 \\
\hline InWingSound & 0.477 & 0.432 & 0.410 \\
\hline ItalyPower & 0.970 & 0.970 & 0.972 \\
\hline LargeKApp & 0.406 & 0.407 & 0.410 \\
\hline Lightning2 & 0.765 & 0.767 & 0.767 \\
\hline Lightning7 & 0.872 & 0.833 & 0.858 \\
\hline MALLLAT & 0.971 & 0.970 & 0.971 \\
\hline Meat & 0.925 & 0.870 & 0.973 \\
\hline MedicalImages & 0.714 & 0.686 & 0.701 \\
\hline MidPhOutlineAgeGrp & 0.507 & 0.347 & 0.445 \\
\hline MidPhOCorrect & 0.823 & 0.821 & 0.819 \\
\hline MidPhTW & 0.329 & 0.314 & 0.320 \\
\hline MoteStrain & 0.925 & 0.920 & 0.915 \\
\hline NonInvECGTh1 & 0.911 & 0.908 & 0.905 \\
\hline NonInvECGTh2 & 0.899 & 0.896 & 0.894 \\
\hline OliveOil & 0.853 & 0.611 & 0.885 \\
\hline OSULeaf & 0.988 & 0.979 & 0.988 \\
\hline PhalOCorrect & 0.812 & 0.803 & 0.809 \\
\hline Phoneme & 0.025 & 0.026 & 0.026 \\
\hline Plane & 0.888 & 0.888 & 0.882 \\
\hline ProxPhOeAgeGrp & 0.600 & 0.594 & 0.436 \\
\hline ProxPhOCorrect & 0.896 & 0.904 & 0.896 \\
\hline ProxPhTW & 0.545 & 0.504 & 0.469 \\
\hline RefDevices & 0.277 & 0.241 & 0.241 \\
\hline ScreenType & 0.297 & 0.302 & 0.308 \\
\hline ShapeletSim & 0.842 & 0.842 & 0.842 \\
\hline ShapesAll & 0.108 & 0.108 & 0.107 \\
\hline SmlKitApp & 0.345 & 0.361 & 0.370 \\
\hline SonyAIBORI & 0.984 & 0.974 & 0.983 \\
\hline SonyAIBORII & 0.980 & 0.978 & 0.977 \\
\hline StarLightCurves & 0.975 & 0.961 & 0.962 \\
\hline Strawberry & 0.818 & 0.818 & 0.818 \\
\hline SwedishLeaf & 0.807 & 0.801 & 0.811 \\
\hline Symbols & 0.980 & 0.982 & 0.974 \\
\hline SynControl & 0.522 & 0.516 & 0.511 \\
\hline ToeSeg1 & 0.708 & 0.746 & 0.746 \\
\hline ToeSeg2 & 0.582 & 0.563 & 0.577 \\
\hline Trace & 1.0 & 0.986 & 0.983 \\
\hline TwoLeadECG & 0.999 & 0.999 & 0.999 \\
\hline TwoPatterns & 0.986 & 0.989 & 0.971 \\
\hline UWaveAll & 0.782 & 0.766 & 0.754 \\
\hline UWaveX & 0.665 & 0.654 & 0.659 \\
\hline UWaveY & 0.698 & 0.695 & 0.686 \\
\hline UWaveZ & 0.736 & 0.739 & 0.743 \\
\hline Wafer & 0.996 & 0.996 & 0.996 \\
\hline Wine & 0.887 & 0.887 & 0.887 \\
\hline WordSynonyms & 0.380 & 0.327 & 0.345 \\
\hline Worms & 0.448 & 0.423 & 0.425 \\
\hline WormsTwoClass & 0.530 & 0.525 & 0.542 \\
\hline Yoga & 0.882 & 0.906 & 0.914 \\
\hline
\end{tabular}


GRU-FCN

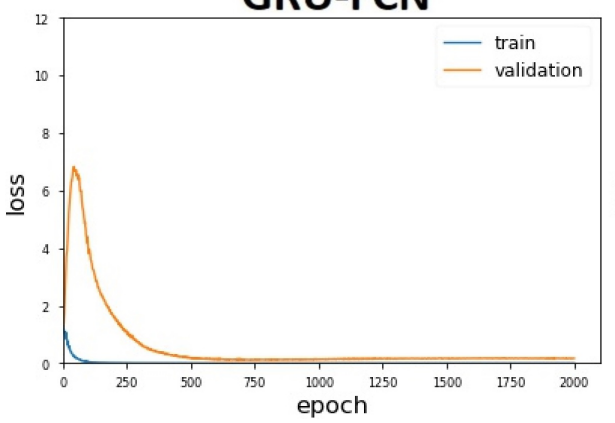

LSTM-FCN

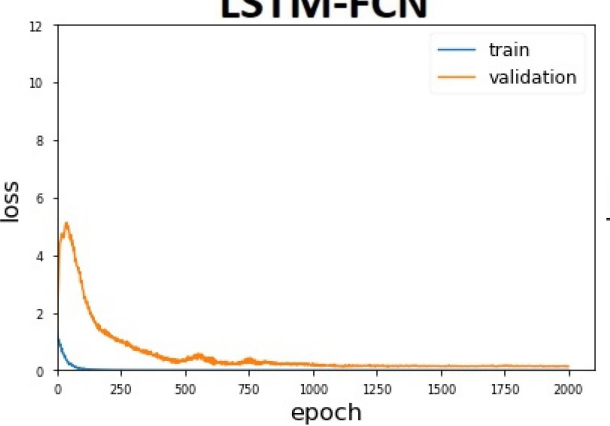

ALSTM-FCN

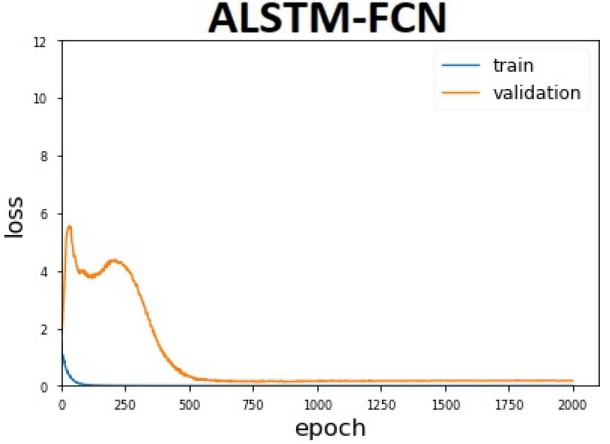

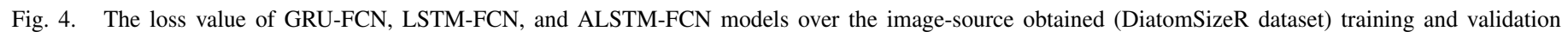
processes.

GRU-FCN

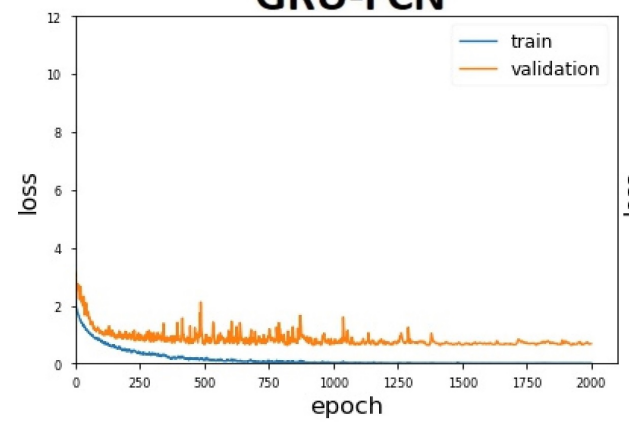

LSTM-FCN

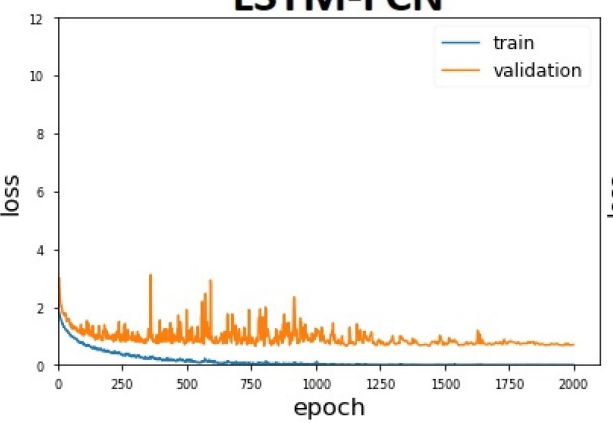

ALSTM-FCN

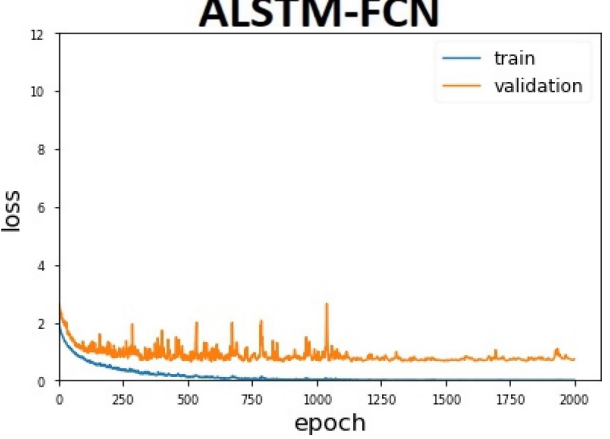

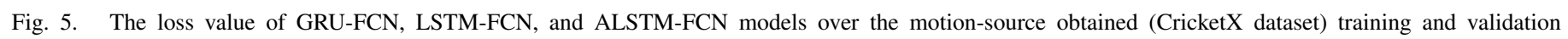
processes.

\section{GRU-FCN}

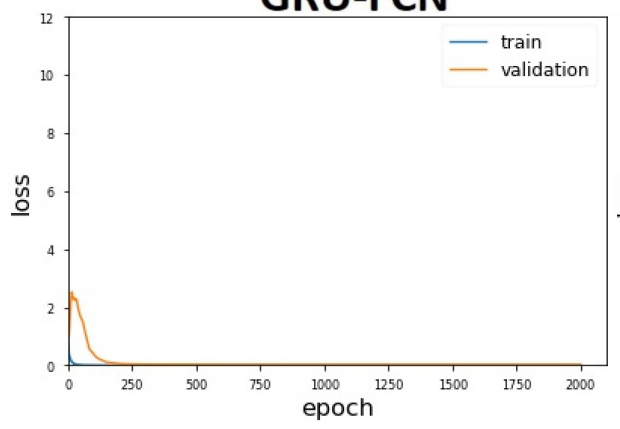

LSTM-FCN

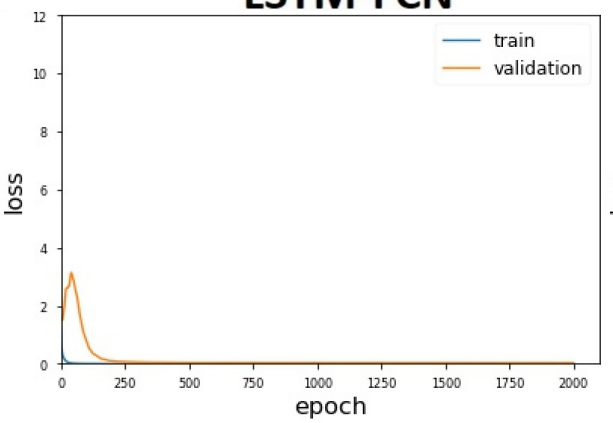

ALSTM-FCN

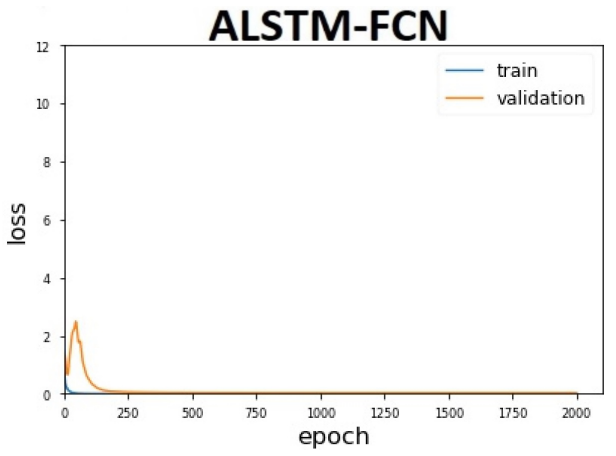

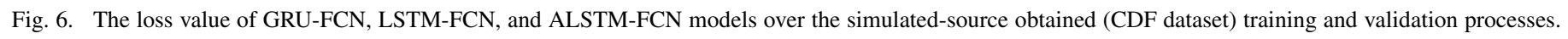
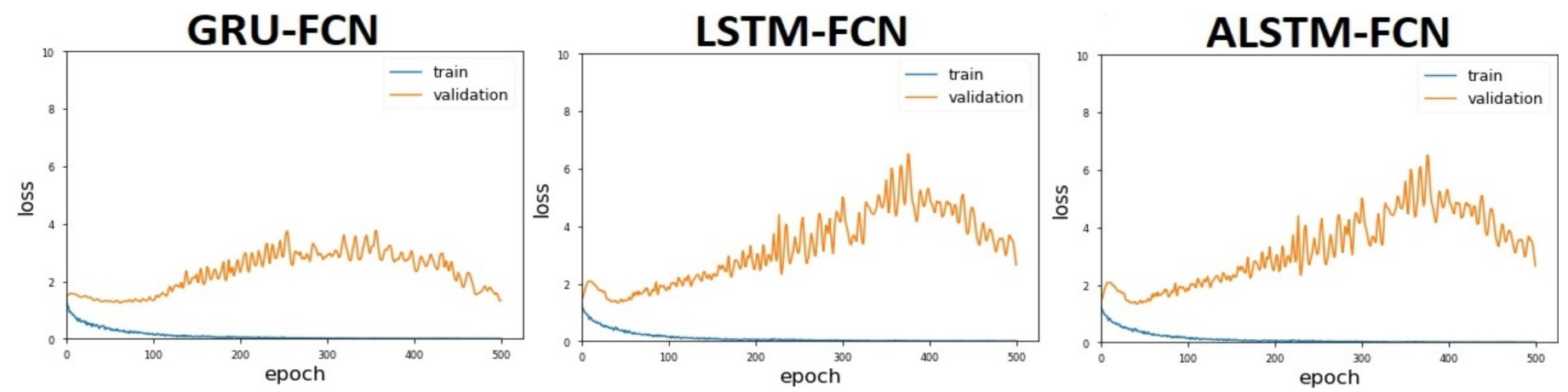

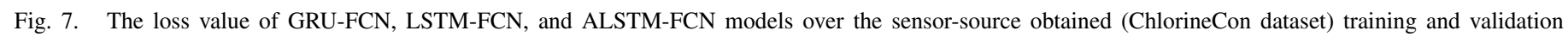
processes. 


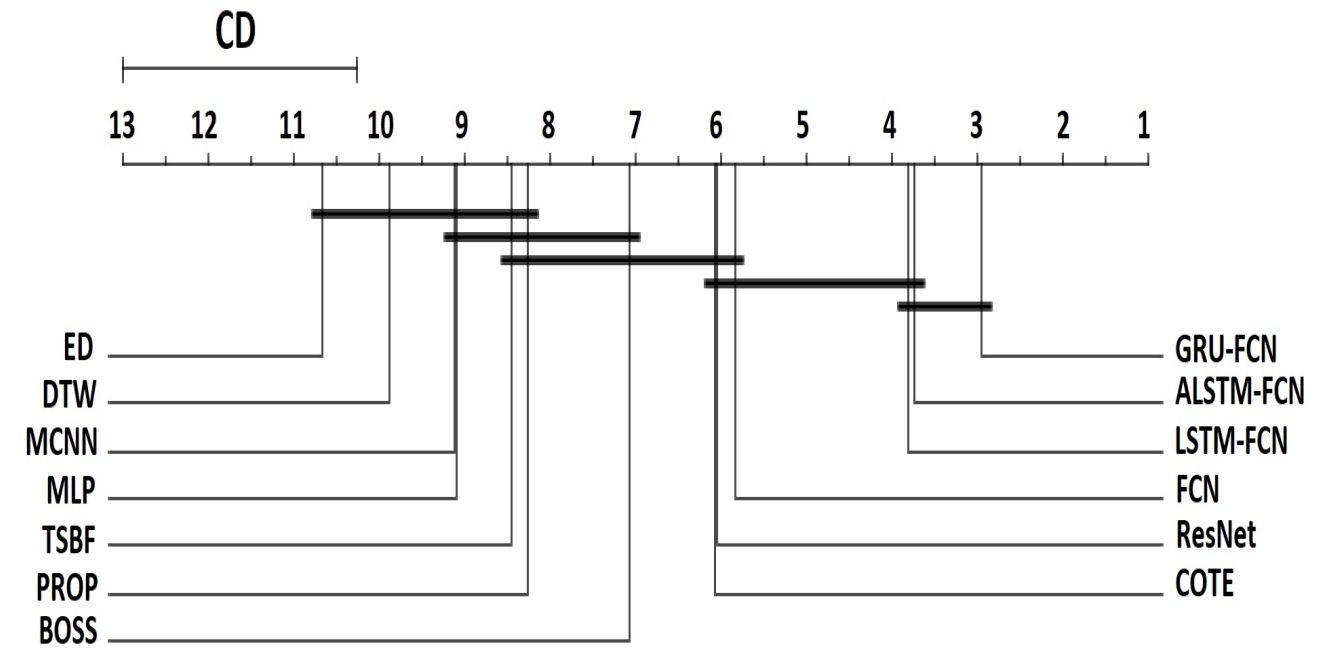

Fig. 8. Critical difference diagram based on the arithmetic mean of model ranks.

TABLE VI. WILCOXON SIGNED-RANK TEST ON GRU-FCN AND 10 BENCHMARK MODEL ON THE 85 DATASETS FROM UCR BENCHMARK [14].

\begin{tabular}{|c|c|c|c|c|c|c|c|c|c|c|c|c|}
\hline & $F C N$ & LSTM-FCN & ALSTM-FCN & ResNet & MCNN & $M L P$ & COTE & DTW & PROP & BOSS & $T S B F$ & $E D$ \\
\hline GRU-FCN & $3.44 \mathrm{E}-10$ & 4.95E-03 & 4.00E-02 & $2.53 \mathrm{E}-11$ & $1.05 \mathrm{E}-12$ & $1.43 \mathrm{E}-13$ & $1.25 \mathrm{E}-08$ & $1.23 \mathrm{E}-14$ & $1.58 \mathrm{E}-11$ & $4.37 \mathrm{E}-10$ & $2.77 \mathrm{E}-12$ & $2.93 \mathrm{E}-15$ \\
\hline FCN & & 4.37E-09 & $8.58 \mathrm{E}-08$ & 1.68E-01 & $9.31 \mathrm{E}-10$ & $1.12 \mathrm{E}-09$ & 1.85E-02 & $3.49 \mathrm{E}-12$ & $1.31 \mathrm{E}-07$ & $8.02 \mathrm{E}-04$ & $1.10 \mathrm{E}-07$ & 7.07E-13 \\
\hline LSTM-FCN & & & $7.45 \mathrm{E}-01$ & $2.24 \mathrm{E}-09$ & $1.40 \mathrm{E}-11$ & $6.09 \mathrm{E}-13$ & $1.03 \mathrm{E}-06$ & $2.35 \mathrm{E}-14$ & $5.72 \mathrm{E}-11$ & $2.85 \mathrm{E}-9$ & $6.40 \mathrm{E}-13$ & $1.08 \mathrm{E}-14$ \\
\hline ALSTM-FCN & & & & 1.40E-07 & $1.02 \mathrm{E}-11$ & $8.35 \mathrm{E}-12$ & 2.33E-07 & $1.55 \mathrm{E}-13$ & $7.95 \mathrm{E}-11$ & 4.71E-09 & $3.30 \mathrm{E}-12$ & $4.73 \mathrm{E}-14$ \\
\hline ResNet & & & & & $6.28 \mathrm{E}-09$ & $1.79 \mathrm{E}-08$ & $2.46 \mathrm{E}-01$ & $9.32 \mathrm{E}-13$ & $1.76 \mathrm{E}-06$ & $1.28 \mathrm{E}-03$ & $1.56 \mathrm{E}-07$ & $1.11 \mathrm{E}-13$ \\
\hline MCNN & & & & & & 4.35E- 05 & 4.77E-08 & $5.76 \mathrm{E}-05$ & 6.10E-04 & $1.20 \mathrm{E}-06$ & $7.72 \mathrm{E}-06$ & 2.18E-04 \\
\hline MLP & & & & & & & 7.04E-05 & $7.28 \mathrm{E}-01$ & 7.13E-01 & $1.08 \mathrm{E}-03$ & $5.70 \mathrm{E}-03$ & $3.25 \mathrm{E}-04$ \\
\hline COTE & & & & & & & & $1.62 \mathrm{E}-06$ & $2.28 \mathrm{E}-05$ & 7.74E-03 & $3.59 \mathrm{E}-04$ & $3.22 \mathrm{E}-07$ \\
\hline DTW & & & & & & & & & 2.05E-01 & 2.37E-07 & $1.80 \mathrm{E}-04$ & 2.13E-03 \\
\hline PROP & & & & & & & & & & 8.82E-03 & 5.13E-01 & 3.14E-02 \\
\hline BOSS & & & & & & & & & & & 3.18E-02 & $7.02 \mathrm{E}-10$ \\
\hline TSBF & & & & & & & & & & & & $6.65 \mathrm{E}-08$ \\
\hline
\end{tabular}

average arithmetic ranking and the lowest mean per-class error (MPCE) through time series datasets classification of the UCR benchmark compared to the state-of-the-art models. Therefore, replacing the LSTM by GRU in the LSTM-FCN for univariate time series classification can improve the classification with smaller model architecture.

\section{ACKNOWLEDGMENT}

We would like to thank Prof. Eamonn Keogh for his suggestions, comments, and ideas about our proposed work in this paper. In addition, we would like to thank the LSTMFCN authors for their implementation availability.

\section{REFERENCES}

[1] J. D. Hamilton, Time series analysis. Princeton University Press, Princeton, NJ, 1994, vol. 2.

[2] H. Sohn and C. R. Farrar, "Damage diagnosis using time series analysis of vibration signals," Smart Materials and Structures, vol. 10, no. 3, p. 446, 2001.

[3] M. Gul and F. N. Catbas, "Statistical pattern recognition for structural health monitoring using time series modeling: theory and experimental verifications," Mechanical Systems and Signal Processing, vol. 23, no. 7, pp. 2192-2204, 2009.

[4] Z. Wang, W. Yan, and T. Oates, "Time series classification from scratch with deep neural networks: a strong baseline," in Neural Networks (IJCNN), 2017 International Joint Conference on. IEEE, 2017, pp. 1578-1585.

[5] F. Karim, S. Majumdar, H. Darabi, and S. Chen, "LSTM fully convolutional networks for time series classification," IEEE Access, vol. 6 , pp. 1662-1669, 2018.
[6] A. Amei, W. Fu, and C.-H. Ho, "Time series analysis for predicting the occurrences of large scale earthquakes," International Journal of Applied Science and Technology, vol. 2, no. 7, 2012.

[7] J. Rotton and J. Frey, "Air pollution, weather, and violent crimes: concomitant time-series analysis of archival data." Journal of Personality and Social Psychology, vol. 49, no. 5, p. 1207, 1985.

[8] E. Keogh and C. A. Ratanamahatana, "Exact indexing of dynamic time warping," Knowledge and Information Systems, vol. 7, no. 3, pp. 358386, 2005.

[9] P. Schäfer, "The BOSS is concerned with time series classification in the presence of noise," Data Mining and Knowledge Discovery, vol. 29, no. 6 , pp. 1505-1530, 2015.

[10] M. G. Baydogan, G. Runger, and E. Tuv, "A bag-of-features framework to classify time series," IEEE Transactions on Pattern Analysis and Machine Intelligence, vol. 35, no. 11, pp. 2796-2802, 2013.

[11] J. Lines and A. Bagnall, "Time series classification with ensembles of elastic distance measures," Data Mining and Knowledge Discovery, vol. 29, no. 3, pp. 565-592, 2015.

[12] A. Bagnall, J. Lines, J. Hills, and A. Bostrom, "Time-series classification with COTE: the collective of transformation-based ensembles," IEEE Transactions on Knowledge and Data Engineering, vol. 27, no. 9, pp. 2522-2535, 2015.

[13] Z. Cui, W. Chen, and Y. Chen, "Multi-scale convolutional neural networks for time series classification," arXiv preprint arXiv:1603.06995, 2016.

[14] Y. Chen, E. Keogh, B. Hu, N. Begum, A. Bagnall, A. Mueen, and G. Batista. (2015, July) The UCR time series classification archive. [Online]. Available: http://www.cs.ucr.edu/ eamonn/time_series_data/

[15] J. Chung, C. Gulcehre, K. Cho, and Y. Bengio, "Empirical evaluation of gated recurrent neural networks on sequence modeling," arXiv preprint arXiv:1412.3555, 2014. 
[16] Y. LeCun, B. Boser, J. S. Denker, D. Henderson, R. E. Howard, W. Hubbard, and L. D. Jackel, "Backpropagation applied to handwritten zip code recognition," Neural Computation, vol. 1, no. 4, pp. 541-551, 1989

[17] Y. LeCun and Y. Bengio, "Convolutional networks for images, speech, and time series," in The Handbook of Brain Theory and Neural Networks. MIT Press, 1995, pp. 255-258.

[18] I. Goodfellow, Y. Bengio, A. Courville, and Y. Bengio, Deep Learning. MIT press Cambridge, 2016, vol. 1.

[19] S. Ioffe and C. Szegedy, "Batch normalization: Accelerating deep network training by reducing internal covariate shift," arXiv preprint arXiv:1502.03167, 2015

[20] V. Nair and G. E. Hinton, "Rectified linear units improve restricted Boltzmann machines," in Proceedings of the 27th International Conference on Machine Learning (ICML-10), 2010, pp. 807-814.

[21] (2019) Keras recurrent layers documentation. [Online]. Available: https://keras.io/layers/recurrent/

[22] Y.-L. Boureau, J. Ponce, and Y. LeCun, "A theoretical analysis of feature pooling in visual recognition," in Proceedings of the 27th international conference on machine learning (ICML-10), 2010, pp. 111-118.

[23] K. He, X. Zhang, S. Ren, and J. Sun, "Delving deep into rectifiers: surpassing human-level performance on imagenet classification," in Proceedings of the IEEE International Conference on Computer Vision, 2015, pp. 1026-1034.

[24] C. Gulcehre, M. Moczulski, M. Denil, and Y. Bengio, "Noisy activation functions," in International Conference on Machine Learning, 2016, pp. 3059-3068.

[25] X. Glorot and Y. Bengio, "Understanding the difficulty of training deep feedforward neural networks," in Proceedings of the Thirteenth International Conference on Artificial Intelligence and Statistics, 2010, pp. 249-256.

[26] F. Chollet et al., "Keras," https://keras.io, 2015.
[27] D. P. Kingma and J. Ba, "Adam: A method for stochastic optimization," arXiv preprint arXiv:1412.6980, 2014.

[28] N. M. Nasrabadi, "Pattern recognition and machine learning," Journal of Electronic Imaging, vol. 16, no. 4, p. 049901, 2007.

[29] M. Abadi, A. Agarwal, P. Barham, E. Brevdo, Z. Chen, C. Citro, G. S. Corrado, A. Davis, J. Dean, M. Devin, S. Ghemawat, I. Goodfellow, A. Harp, G. Irving, M. Isard, Y. Jia, R. Jozefowicz, L. Kaiser, M. Kudlur, J. Levenberg, D. Mané, R. Monga, S. Moore, D. Murray, C. Olah, M. Schuster, J. Shlens, B. Steiner, I. Sutskever, K. Talwar, P. Tucker, V. Vanhoucke, V. Vasudevan, F. Viégas, O. Vinyals, P. Warden, M. Wattenberg, M. Wicke, Y. Yu, and X. Zheng, "TensorFlow: Large-scale machine learning on heterogeneous systems," 2015, software available from tensorflow.org. [Online]. Available: https://www.tensorflow.org/

[30] Y.-S. Jeong, M. K. Jeong, and O. A. Omitaomu, "Weighted dynamic time warping for time series classification," Pattern Recognition, vol. 44, no. 9, pp. 2231-2240, 2011.

[31] Y. Sasaki et al., "The truth of the f-measure," Teach Tutor mater, vol. 1, no. 5, pp. 1-5, 2007.

[32] D. M. Powers, "Evaluation: from precision, recall and f-measure to roc, informedness, markedness and correlation," 2011.

[33] J. Demšar, "Statistical comparisons of classifiers over multiple data sets," Journal of Machine Learning Research, vol. 7, no. Jan, pp. 1-30, 2006.

[34] T. Pohlert, "The pairwise multiple comparison of mean ranks package (PMCMR)," R Package, vol. 27, 2014.

[35] R. Woolson, "Wilcoxon signed-rank test," Wiley encyclopedia of clinical trials, pp. 1-3, 2007.

[36] D. Rey and M. Neuhäuser, "Wilcoxon-signed-rank test," International encyclopedia of statistical science, pp. 1658-1659, 2011.

[37] R. Lowry, "Concepts and applications of inferential statistics," 2014. 